OPEN ACCESS

Edited by:

Xuelei Ma,

Sichuan University, China

Reviewed by:

Asaf Maoz,

Dana-Farber Cancer Institute,

United States

Martina Spiljar,

University of Geneva, Switzerland

*Correspondence:

Jianfeng Zhou

ZhouJF@pumch.cn

Specialty section:

This article was submitted to

Cancer Immunity

and Immunotherapy,

a section of the journal

Frontiers in Oncology

Received: 04 November 2020 Accepted: 05 January 2021

Published: 22 February 2021

Citation:

Tang H, Zhou J and Bai C (2021) The Efficacy and Safety of Immune

Checkpoint Inhibitors in Patients

With Cancer and Preexisting

Autoimmune Disease.

Front. Oncol. 11:625872.

doi: 10.3389/fonc.2021.625872

\section{The Efficacy and Safety of Immune Checkpoint Inhibitors in Patients With Cancer and Preexisting Autoimmune Disease}

\author{
Hui Tang, Jianfeng Zhou* and Chunmei Bai \\ Department of Medical Oncology, Peking Union Medical College Hospital, Chinese Academy of Medical Sciences and Peking Union \\ Medical College, Beijing, China
}

Immune checkpoint inhibitor $(\mathrm{ICI})$ is a revolutionary breakthrough in the field of cancer treatment. Because of dysregulated activation of the immune system, patients with autoimmune disease (AID) are usually excluded from $\mathrm{ICl}$ clinical trials. Due to a large number of cancer patients with preexisting AID, the safety and efficacy of ICls in these patients deserve more attention. This review summarizes and analyzes the data regarding $\mathrm{ICl}$ therapy in cancer patients with preexisting AID from 17 published studies. Available data suggests that the efficacy of ICls in AID patients is comparable to that in the general population, and the incidence of immune-related adverse events (irAEs) is higher but still manageable. It is recommended to administer ICls with close monitoring of irAEs in patients with a possibly high benefit-risk ratio after a multidisciplinary discussion based on the patient's AID category and severity, the patient's tumor type and prognosis, alternative treatment options, and the patient's intention. Besides, the prevention and management of irAEs in AID patients have been discussed.

Keywords: autoimmune disorder, solid tumors, immunotherapy, immune-related adverse events, PD-1, CTLA-4

\section{INTRODUCTION}

The approved immune checkpoint inhibitors (ICIs) mainly involve several immune checkpointdirected antibodies targeting cytotoxic T lymphocyte-associated antigen 4 (CTLA-4) and programmed cell death protein 1 (PD-1) or its ligand, programmed cell death ligand 1 (PD-L1). CTLA-4 inhibits an immune response in several ways, including hindering autoreactive T-cell activation at a proximal step in the immune response, typically in lymph nodes $(1,2)$. In contrast, the PD-1 pathway regulates $\mathrm{T}$ cells at a later stage of the immune response, typically in peripheral tissues (3). Differing from traditional chemotherapy and targeted therapy, ICI can break the state of immune tolerance in the tumor microenvironment (TME) and activate the body's anti-tumor immunity. Clinical trials of ICI therapy are in full swing, showing remarkable efficacy as well as fewer and milder adverse events compared to chemotherapy, and the indications for ICIs continue to expand across malignancies (4).

Autoimmune diseases (AIDs) represent a family of at least 80 diseases that share a common pathogenesis: an improper activation of the immune system attacking the body's own organs (5). 
By increasing the activity of the immune system, ICI may result in immune-related adverse events (irAEs). Although the precise underlying mechanism is unknown, irAEs show many common clinical manifestations and pathophysiology features similar to AID (6). The role of PD-1 and CTLA-4 in autoantigen tolerance has been widely recognized (7), so ICI may exacerbate the damage done by one's own immune system to AID patients and bring forth autoimmune inflammatory manifestations similar to or unrelated to the baseline AID. AID patients were usually excluded from clinical trials of ICI therapy due to the potential for increased toxicity (8).

AIDs may cause chronic inflammation and increase the risk of carcinogenesis (9-12). Cancer can also increase the risk of AIDs (13). The incidence of AIDs in lung cancer patients was reported to be as high as $14 \%$ to $25 \%$ (8), and some cancers show clinical features similar to AIDs $(9,14)$. Due to a large number of cancer patients with preexisting AID, the safety and efficacy of ICIs in these patients deserve more attention. Furthermore, there is a tendency toward immune activation in AID patients, so some clinicians proposed that AID patients may be likely to benefit more from ICI (15). At present, there is no reported prospective randomized controlled study on this issue. Whether the efficacy and safety of ICIs in patients with cancer and preexisting AID differ from that in the general population is unclear.

\section{RELATIONSHIP BETWEEN IMMUNE CHECKPOINTS AND AID}

CTLA-4 signaling has been shown to be involved in the pathogenesis of many AIDs including rheumatoid arthritis (RA), systemic lupus erythematosus (SLE), multiple sclerosis (MS), and type-1 diabetes (T1D) (16). Polymorphisms in CTLA4 gene were also associated with disease susceptibility to many AIDs like SLE, RA, MS, T1D, and so on (1). Human patients with heterozygous loss of function mutations in CTLA-4 developed widespread autoimmunity including autoimmune hepatitis, T1D, and arthritis (17). CTLA-4 gene deletion could lead mice to develop lymphoproliferative disease and die by 3-4 weeks of age (18). By contrast, mice with ablation of CTLA-4 expression during adulthood developed severe, but not fatal, autoimmunity (19). Preclinical models showed CTLA-4 blockade worsened autoimmune thyroiditis, with a more aggressive mononuclear cells infiltration in thyroid (20).

PD-1 signaling were also involved in the pathogenesis of many AIDs like autoimmune hepatitis, inflammatory bowel disease (IBD), SLE, myocarditis, and RA (21). Polymorphisms in PDCD1 gene were associated with disease susceptibility to many AIDs including SLE, RA, Graves' disease, and so on (1). The engagement of Tregs with autoreactive B cells via the PD-1/ L1 inhibitory axis can trigger B cells apoptosis and inhibit the production of autoantibody (22). In patients with RA, lymphocytes infiltrating the synovium commonly express $\mathrm{PD}$ 1 , the synovial lining cells express PD-L1, and the number of PD1-positive lymphocytes was significantly larger in RA than in osteoarthritis (23). In addition, the PD-L1 expression on synovial lining cells was positively related to the number of infiltrating $\mathrm{T}$ cells and Krenn's synovitis score (23), indicating an important role of PD-1 pathway in RA. Notably, in non-obese diabetic mice, both anti-CTLA-4 and anti-PD-1 treatment can prevent anergy induction in islet antigen-specific T cells, but only PD-1/ L1 blockade can reverse experimentally induced anergy, indicating a unique function for PD-1 signaling in maintaining T cells anergy (24).

Furthermore, the administration of ICI may conflict with the management of AIDs. For example, abatacept is a fusion protein comprising the extracellular domain of CTLA-4, that competitively blocks the T cells CD28-CD80 pathway signaling and improves the prognosis of RA (25). Therefore, in contrast, the use of ipilimumab which blockades CTLA-4 signaling may conflict with the management of RA.

\section{LITERATURE EXPERIENCE WITH ICls IN AID PATIENTS}

To evaluate the efficacy and safety of ICIs in patients with cancer and preexisting AID, we summarized the retrospective studies published before October 2020 (Table 1). Inclusion criteria was articles available in full text, published in English, and reporting safety or efficacy data on patients with preexisting AID and cancer treated with ICI. Further, case reports and review articles were excluded. After screening, 17 published studies were included, from which the following data were extracted: author, publication year, sample size, characteristics of AID, cancer and ICI type; the number and proportion of AID flares, newly developed irAEs, treatment discontinuation and response; survival time. The irAEs reported in these studies can be divided into two categories. The first type is the flare of preexisting AIDs, and the second type is the newly developed irAEs that does not have a clear causal link with preexisting AIDs. We refer to the two types collectively as total irAEs (TirAEs). Most studies included patients regardless of the treatment line, so caution should be exercised when comparing studies' efficacy data with previous clinical trials.

\section{Efficacy and Safety of ICls in Patients With Melanoma and AID}

Rich experience has been accumulated in ICI therapy for malignant melanoma. There were three retrospective studies of anti-CTLA-4 therapy for melanoma patients with AID (26-28) (Table 1). Two studies reported that the objective response rate (ORR) was no more than $20 \%(26,27)$, and another study, with just eight patients, reported an ORR as high as 50\% (28). As to the safety, Johnson et al. (27) reported that the incidence of TirAEs in AID patients was 50\%, including an incidence rate of AID flare of $27 \%$. Kähler et al. (26) reported that the incidence of TirAEs in AID patients was $44 \%$. The incidence of both AID flare and newly developed irAEs were $29 \%$, and $17 \%$ of patients discontinued ICIs because of AID flare. Both studies $(26,27)$ supported that the incidence of TirAEs in AID patients did not exceed that observed in the general population included in 
TABLE 1 | Data summary of cancer patients with preexisting autoimmune disease treated with immune checkpoint inhibitors.

\begin{tabular}{|c|c|c|c|c|c|c|c|c|c|c|c|}
\hline \multirow[t]{2}{*}{ Cancer } & \multirow[t]{2}{*}{ Target } & \multirow[t]{2}{*}{ Study } & \multirow[t]{2}{*}{ Main AID } & \multirow[t]{2}{*}{$\mathbf{N}$} & \multicolumn{3}{|c|}{ Efficacy } & \multicolumn{4}{|c|}{ Safety } \\
\hline & & & & & ORR & $\begin{array}{c}\text { mPFS } \\
\text { (months) }\end{array}$ & $\begin{array}{c}\text { mOS } \\
\text { (months) }\end{array}$ & $\begin{array}{c}\text { TirAEs } \\
\text { (grade } \geq 3 \text { ) }\end{array}$ & $\begin{array}{l}\text { Preexisting AID } \\
\text { flare (grade } \geq 3 \text { ) }\end{array}$ & $\begin{array}{c}\text { Newly } \\
\text { developed irAEs } \\
\text { (grade } \geq 3 \text { ) }\end{array}$ & Treatment discontinuation \\
\hline Melanoma & CTLA-4 & Kähler (26) & $\begin{array}{l}\text { Thyroiditis (37\%), Ps (17\%), RA } \\
(15 \%)\end{array}$ & 41 & $12 \%$ & NA & NA & $44 \%(N A)$ & $29 \%(N A)$ & $29 \%(N A)$ & $17 \%$ due to AID flare \\
\hline Melanoma & CTLA-4 & Johnson (27) & RA (20\%), IBD (20\%), Ps (17\%) & 30 & $20 \%$ & 3.0 & 12.5 & $50 \%(N A)$ & $27 \%(N A)$ & $33 \%(33 \%)$ & NA \\
\hline Melanoma & CTLA-4 & Lee (28) & RA (100\%) & 8 & $50 \%$ & NA & NA & $100 \%(63 \%)$ & $75 \%(25 \%)$ & $50 \%(50 \%)$ & $63 \%$ due to TirAEs \\
\hline Melanoma & PD-1 & Menzies (15) & RA (25\%), Ps (12\%), Colitis (10\%) & 52 & $33 \%$ & 6.2 & NR & NA & $38 \%(6 \%)$ & $29 \%(10 \%)$ & $\begin{array}{l}4 \% \text { and } 8 \% \text { permanent discontinuation due to } \\
\text { AID flare and newly developed irAEs, } \\
\text { respectively }\end{array}$ \\
\hline Melanoma & PD-1 & Gutzmer (29) & $\begin{array}{l}\text { Thyroiditis (26\%), RA (21\%), Ps } \\
(16 \%)\end{array}$ & 19 & $32 \%$ & NA & NA & $58 \%(16 \%)$ & $42 \%(11 \%)$ & $16 \%(5 \%)$ & None \\
\hline NSCLC & PD-1 & Yoneshima (30) & ANA positivity (100\%) & 18 & $28 \%$ & 2.9 & 11.6 & $33 \%(11 \%)$ & NA & NA & $11 \%$ due to TirAEs \\
\hline NSCLC & PD-1 & Leonardi (31) & $\begin{array}{l}\text { Ps }(25 \%), \text { RA (20\%), Thyroiditis } \\
(16 \%)\end{array}$ & 56 & $22 \%$ & NA & NA & $55 \%(N A)$ & $23 \%(4 \%)$ & $38 \%(11 \%)$ & $\begin{array}{l}0 \% \text { and } 14 \% \text { permanent discontinuation due to } \\
\text { AID flare and newly developed irAEs, } \\
\text { respectively }\end{array}$ \\
\hline $\begin{array}{l}\text { Urological } \\
\text { cancers }\end{array}$ & PD-1 & Loriot (32) & $\begin{array}{l}\text { Ps (43\%), Thyroiditis (17\%), RA } \\
(11 \%)\end{array}$ & 35 & $11 \%$ & 8.2 & 4.4 & $46 \%(14 \%)$ & $11 \%(6 \%)$ & NA & $14 \%$ due to TirAEs \\
\hline $\begin{array}{l}\text { Urological } \\
\text { cancers }\end{array}$ & $\begin{array}{l}\text { PD-1 or } \\
\text { CTLA-4 }\end{array}$ & $\begin{array}{l}\text { Martinez } \\
\text { Chanza (33) }\end{array}$ & $\begin{array}{l}\text { Ps (23\%), Thyroiditis (13\%), RA } \\
(11 \%)\end{array}$ & 106 & $35 \%$ & NA & NA & $58 \%(N A)$ & $36 \%(6 \%)$ & $38 \%(12 \%)$ & $\begin{array}{l}6 \% \text { and } 8 \% \text { permanent discontinuation due to } \\
\text { AID flare and newly developed irAEs, } \\
\text { respectively }\end{array}$ \\
\hline Various & $\mathrm{PD}-1$ & Danlos (34) & $\begin{array}{l}\text { Vitiligo (38\%), Ps (27\%), } \\
\text { Thyroiditis (16\%) }\end{array}$ & 45 & $38 \%$ & NA & NA & $44 \%(11 \%)$ & $24 \%(N A)$ & $22 \%(N A)$ & $11 \%$ due to TirAEs \\
\hline Various & PD-1 & Cortellini (35) & Ps (40\%), RA (27\%), IBD (13\%) & $15^{\mathrm{a}}$ & $50 \%$ & 6.8 & 9.8 & $73 \%(13 \%)$ & $47 \%(13 \%)$ & NA & $13 \%$ due to TirAEs \\
\hline & & & $\begin{array}{l}\text { Thyroiditis (76\%), Ps (10\%), } \\
\text { Vitiligo (3\%) }\end{array}$ & $70^{\mathrm{b}}$ & $38 \%$ & 14.4 & 15.7 & $64 \%(9 \%)$ & $47 \%(9 \%)$ & NA & $6 \%$ due to TirAEs \\
\hline Various & $\begin{array}{l}\text { PD-1 or } \\
\text { CTLA-4 }\end{array}$ & Tison (36) & Ps (28\%), RA (18\%), IBD (13\%) & 112 & $49 \%^{\mathrm{c}}$ & NA & NA & $71 \%(N A)$ & $47 \%(13 \%)$ & $42 \%(16 \%)$ & $21 \%$ permanent discontinuation due to TirAEs \\
\hline Various & $\begin{array}{l}\text { PD-1 or } \\
\text { CTLA-4 }\end{array}$ & Richter (37) & $\begin{array}{l}\text { RA }(31 \%) \text {, Polymyalgia } \\
\text { rheumatica }(31 \%) \text {, Sjogren's } \\
\text { syndrome }(13 \%)\end{array}$ & 16 & NA & NA & NA & $38 \%(25 \%)$ & $6 \%(0 \%)$ & $31 \%(25 \%)$ & $38 \%$ due to TirAEs \\
\hline Various & $\begin{array}{l}\text { PD-1 or } \\
\text { CTLA-4 }\end{array}$ & Kaur (38) & $\begin{array}{l}\text { Hypothyroidism (61\%), RA (9\%), } \\
\text { Ps }(9 \%)\end{array}$ & 46 & NA & NA & NA & NA & $20 \%(N A)$ & NA & $2 \%$ due to AID flare \\
\hline Various & $\begin{array}{l}\text { PD-1 or } \\
\text { CTLA-4 }\end{array}$ & Abu-Sbeih (39) & $\mathrm{IBD}(100 \%)$ & 102 & NA & NA & NA & NA & $36 \%(17 \%)$ & NA & $32 \%$ due to TirAEs \\
\hline Various & $\begin{array}{l}\text { PD-1 or } \\
\text { CTLA-4 }\end{array}$ & Braga Neto (40) & $\mathrm{IBD}(100 \%)$ & 13 & NA & NA & NA & NA & $31 \%(N A)$ & NA & None \\
\hline Various & $\begin{array}{l}\text { PD-1 or } \\
\text { CTLA-4 }\end{array}$ & Efuni (41) & RA (100\%) & 22 & NA & NA & NA & $73 \%$ & $55 \%$ & $32 \%(9 \%)$ & $\begin{array}{l}9 \% \text { and } 5 \% \text { permanent discontinuation due to } \\
\text { AID flare and newly developed irAEs, } \\
\text { respectively }\end{array}$ \\
\hline
\end{tabular}

cIn patients without prior ICl therapy. 
previous large clinical trials $(42,43)$. Furthermore, AID flares and newly developed irAEs were usually manageable according to established algorithms (44-46), mostly with corticosteroids or other immunosuppressants, and did not preclude clinical benefit.

There were also two retrospective studies $(15,29)$ on anti-PD1 therapy for melanoma patients with AID (Table 1). Both Menzies et al. (15) and Gutzmer et al. (29) reported that the ORR of anti-PD-1 antibodies in melanoma patients with preexisting AID exceeded $30 \%$. Intriguingly, large clinical trials demonstrated that ORR of anti-PD-1 therapy in melanoma was $33 \%$ to $45 \%$ in the first-line treatment $(42,43,47)$ and $21 \%$ to $32 \%$ after ipilimumab $(48,49)$. Considering the high prevalence of adverse prognostic features (such as 31\% of patients had brain metastases, $48 \%$ of patients had elevated serum $\mathrm{LDH}$, and $56 \%$ of patients' ECOG grade were $\geq 1$ ) and $54 \%$ of patients had previous anti-CTLA-4 therapy in the included patients, Menzies et al. (15) speculated that patients with a tendency toward autoimmunity might be more likely to benefit from anti-PD-1 therapy. Taken together, the above two studies $(15,29)$ suggested that the efficacy of anti-PD-1 therapy in patients with melanoma and AID was not inferior to that in the general population. As to the safety, Gutzmer et al. (29) reported that the incidence of any grade and grade 3/4 TirAEs were as high as $58 \%$ and $16 \%$, respectively. The discontinuation rates of anti-PD-1 therapy in the study of Menzies et al. (15) were comparable to those in general melanoma patients $(42,43,47)$, and the immune damage of AID flare was generally mild and manageable.

\section{Efficacy and Safety of ICls in Patients With Other Cancers and AID}

There are relatively few studies on ICI therapy in patients with non-small cell lung cancer (NSCLC) and preexisting AID. Yoneshima et al. (30) found that antinuclear antibody (ANA) positivity had no significant effect on ORR and the incidence of irAEs in NSCLC patients treated with anti-PD-1, but both the median progression-free survival (mPFS) and median overall survival (mOS) were significantly shorter in ANA-positive patients than in ANA-negative patients (2.9 versus 3.8 months, 11.6 versus 15.8 months, $p=0.03$ for each instance). Moreover, the authors also identified three patients with increased ANA titer during the anti-PD-1 treatment, all of whom subsequently developed irAEs. The study of Leonardi et al. (31) demonstrated that the ORR was $22 \%$ in NSCLC patients with AID, the incidence of TirAEs was 55\%, and the safety was comparable to that in the general population.

There were 141 patients with urological cancers and AID in the studies reported by Martinez Chanza et al. (33) and Loriot et al. (32). The most common preexisting AIDs were psoriasis (Ps, $n=39)$, thyroiditis $(n=30)$, and RA $(n=16)$. In the studies reported by Martinez Chanza et al. (33), the rates of AID flare and newly developed irAEs were as high as $36 \%$ and $38 \%$, respectively. However, TirAEs in the above two studies $(58 \%$ and $46 \%$, respectively) were generally mild and reversible, especially in patients with asymptomatic or mildly symptomatic AID, and the efficacy was similar in AID and non-AID patients. As to AIDs of clinical concern, such as Guillain-Barre syndrome (GBS), MS, and IBD, flares did not appear more frequent but might be more aggressive as most of them resulted in ICI discontinuation.

In the studies on the use of ICIs in cancer patients with unlimited tumor types and preexisting AID, the majority of malignant tumor types were still melanoma and/or NSCLC (Table 1) (34-40) Danlos et al. (34) analyzed data from a large prospective study of anti-PD-1 treatment and found that the 45 patients with AID had no significant difference in ORR or mOS compared with those without AID, but the median irAE-free survival time was significantly shorter (5.4 versus 13.0 months, $p=0.0002)$ and the incidence of TirAEs was higher in patients with AID (44\% versus 29\%). Tison et al. (36) reported a large multicenter retrospective study including 112 AID patients treated for various cancers with ICIs. Forty-nine percent of 105 patients without prior ICI therapy were considered to be responders. After a median follow-up period of 8 months, the mPFS of melanoma and NSCLC patients was 12.9 months and 11.8 months, respectively, and the mOS was not reached and 22.4 months. However, the incidences of TirAEs and permanent treatment discontinuation $(71 \%$ and $21 \%$, respectively) were higher than that of the general population, which needs to be noticed. In the study of Cortellini et al. (35), the ORR of anti-PD1 treatment in 85 cancer patients with AID was $40 \%$. Unfortunately, the incidence of TirAEs in both inactive and active AID patients were significantly higher than that of the general population ( $64 \%$ versus $40 \%, p=0.0005$, and $73 \%$ versus $40 \%, p=0.0162$, respectively). The incidence of TirAEs is alarming and implies that clinicians should be vigilant when using ICIs in AID patients, especially in those with active AID. Nevertheless, the AID comorbidity in this study was not significantly related to the incidence of grade $3 / 4$ irAEs, treatment discontinuation rate, ORR, PFS, or OS.

Summarily, the above studies tend to support the conclusion that the efficacy of ICIs in patients with AID is comparable to that in the general population, while the incidence of TirAEs is higher, most TirAEs are mild and manageable.

\section{The Effect of AID Status and Immunosuppressive Therapy on Efficacy and Safety of ICls}

It is controversial whether the use of glucocorticoids at a daily dose of $10 \mathrm{mg}$, or more of prednisone or other immunosuppressants, at the beginning of ICI therapy or within 1 month would affect the efficacy of ICI $(50,51)$. In the study of Kähler et al. (26), 11 of the 41 patients were undergoing at least one systemic immunosuppression at the time of ipilimumab initiation, including eight patients receiving a low-dose prednisone. The result suggested that patients receiving immunosuppressive therapy had a similar ORR of $9 \%$ compared to patients without immunosuppressive therapy. The studies of Gutzmer et al. (29) and Leonardi et al. (31) also supported that the use of immunosuppressants at the beginning of treatment does not affect the efficacy of ICI. However, in the study 
reported by Menzies et al. (15), 20 of the 52 patients with AID were on systemic immunosuppression at the start of anti-PD-1 therapy, including 14 patients receiving corticosteroids. The result showed that the ORR in the patients undergoing immunosuppressive therapy was significantly lower than in those not undergoing immunosuppressive therapy ( $15 \%$ versus $44 \%, p=0.033$ ). The meta-analysis of Xie et al. (52) also demonstrated that there was a trend toward lower ORR in patients with immunosuppressants at ICI start $(p>0.05)$. However, due to the heterogeneity of included patients, the data on the pooled ORR may not be so meaningful. As for survival data, Tison et al. (36) reported that the 51 patients receiving immunosuppressive therapy had a shorter mPFS than those without immunosuppressive agents ( 3.8 versus 12 months, $p=$ 0.006), but the mOS difference was not significant. Furthermore, Cortellini et al. (35) reported that the patients with active AID tended to have a higher ORR but shorter mPFS and mOS when compared with patients with inactive AID.

On the aspect of safety, Menzies et al. (15) reported that flares occurred more often in patients with active AID than in those with inactive AID (60\% versus $30 \%, p=0.039$ ), and there was a trend for more flares in patients on immunosuppression treatment than in those not on immunosuppression $(50 \%$ versus $31 \%, p>0.05$ ). The study of Martinez Chanza et al. (33) also reached a conclusion consistent with that of Menzies et al. Similarly, the study of Leonardi et al. (31) suggested that AID was more likely to flare in patients with active symptoms, and Kähler et al. (26) also reported that flares occurred more often in patients on immunosuppression at the start of treatment. However, Abu-Sbeih et al. (39) reported that there was no significant correlation between active IBD within 3 months before ICIs initiation and all grades of gastrointestinal (GI) adverse events, but patients with active IBD had more severe GI adverse events. The meta-analysis of Xie et al. (52) reported that the pooled incidence of AID flare and newly developed irAEs were $35 \%$ and $33 \%$, respectively, and there was no statistical difference between patients with and without immunosuppressive therapy regarding AID flare. Besides, almost all AID flares occurred at the beginning of ICI therapy2 weeks to 1 year after initiation, but mostly around 1-2 months $(15,27,29,31,33,41)$. Most AID flares had similar manifestations and affected the same anatomic sites as prior AID symptoms $(27,31,36)$.

Summarily, available studies mainly supported that immunosuppressive therapy controlling AID might have little influence on the efficacy of ICIs, but the incidence of TirAEs or AID flare would be higher in patients with active AID or undergoing immunosuppressive therapy. Furthermore, it is important to note that there are few patients with severe active AID included in the retrospective studies. It may be necessary to properly treat and control severe active AID before ICI initiation for safety's sake.

\section{The Effect of AID and ICI Category on the Safety of ICls}

Almost all common AIDs have been included in the retrospective studies. However, some AIDs (such as neurological AIDs) will result in devastating consequences if they flare, so clinicians would rarely use ICIs in patients with those AIDs. Therefore, patients with those AIDs may be underrepresented in the retrospective studies. Many studies yielded similar conclusions $(15,26-29,31,33-36,38)$. That is, preexisting rheumatologic AIDs (such as RA, polymyalgia rheumatica) and Ps were most likely to effect AID flare after ICI therapy (the incidences of AID flare were $50 \%$ to $68 \%$ and $20 \%$ to $67 \%$ in patients with rheumatologic AIDs and Ps, respectively). This is in line with the review of Ramos-Casals et al. (53) and the meta-analysis of Xie et al. (52). Most of the studies suggested that autoimmune thyroiditis, GI AIDs (such as IBD/colitis), neurological AIDs, and respiratory AIDs (such as asthma) were less likely to effect AID flare $(15,26,29,31,33,34)$. Part of the possible reason for this is that the pathogenesis of various AIDs is heterogeneous. The PD-1/L1 pathway plays an important role in rheumatism, while many other AIDs do not involve or rely heavily on the PD-1-signaling pathway (15). For example, GBS is a typical B-cell-mediated disease, while chronic inflammation of RA is characterized by $\mathrm{PD}$-1-positive T-cell infiltration $(54,55)$. However, due to a higher mortality rate in neurologic irAEs, NCCN guidelines had recommended more aggressive measures to neurological irAEs (46). Therefore, ICIs should be used more cautiously in patients with preexisting neurological AID.

As to ICI category, Tison et al. (36) reported that newly developed irAEs appeared to be more frequent (11/14 [79\%] versus $34 / 95$ [36\%]) and severe in the ipilimumab group than in the anti-PD-1 therapy group. This is consistent with the conclusion that the incidence and severity of irAEs induced by anti-CTLA-4 treatment were higher or severer than that of antiPD-1 treatment in the general population (56). This is also in line with severe autoimmunity and lymphoproliferative disorder observed in CTLA-4 gene deletion mice $(18,19)$ compared to moderate autoimmunity, including aplastic anemia, glomerulonephritis, and arthritis, seen in PD-1-deficient mice (57, 58). Colitis and hypophysitis were two common ipilimumab-induced irAEs in AID patients $(26-28,37)$. It is still not entirely clear why ICIs at different targets could produce organ-specific irAEs, and CTLA-4 expression on normal pituitary cells might explain hypophysitis induced by antiCTLA-4 therapy $(59,60)$.

Colitis is a particularly frequent and potentially fatal ipilimumab-induced irAE, so the use of ipilimumab in IBD patients was of particular clinical interest. The study reported by Abu-Sbeih et al. (39) enrolled 102 IBD patients treated with ICIs, 17 of those patients receiving ipilimumab. The results showed that anti-CTLA-4 therapy and IBD involving the colon before ICIs initiation were possible risk factors for GI toxicities. However, no GI adverse event-related death was reported, and the response to ICIs in patients with underlying IBD was comparable to that in non-IBD patients, indicating the potential clinical benefit. The study reported by Johnson et al. (27) included six asymptomatic or minimally symptomatic IBD patients receiving ipilimumab. Three patients had prior colectomies, and the other three patients were receiving 
aminosalicylate derivatives or topical hydrocortisone at the time of ipilimumab initiation. After ipilimumab treatment, one patient with ulcerative colitis developed an AID flare and another patient with Crohn disease had newly developed ipilimumab-induced colitis; these were resolved by infliximab and corticosteroids, respectively. There were three IBD patients receiving ipilimumab in the study of Kähler et al. (26), and two of them were receiving mesalazine at the time of ipilimumab initiation. Only one patient with ulcerative colitis developed an AID flare, and this was resolved after treatment discontinuation and prednisone pulse therapy. Therefore, we suggest that ipilimumab might be considered for cancer patients with IBD, if necessary, provided that IBD status is stable and closely monitored.

A systematic review conducted by Abdel-Wahab et al. (61) in 2018 included 123 patients with preexisting AID receiving ICIs. The results demonstrated that no differences in irAEs were observed in patients with active versus inactive AID, and patients receiving immunosuppressive therapy at the time of ICIs initiation appeared to have fewer irAEs. In this systematic review, the incidence of AID flare induced by anti-PD-1 was higher than that by ipilimumab, and more newly developed irAEs were reported with ipilimumab. Some conclusions reached by this systematic review were contradictory to other reviews (6, 56), including ours. This may be partly due to the small number of cases and the variety of AID included in the study of AbdelWahab et al., so prospective studies are needed to determine the safety and efficacy of ICIs in patients with AID (6).

\section{STRATEGIES TO PREVENT AND MANAGE irAEs IN AID PATIENTS}

Immunotherapy-induced inflammation and tumor lysis generate numerous antigens that can be presented by antigen-presenting cells and trigger a secondary immune response to autoantigen. This process is defined as epitope spreading, which plays a crucial role in the initial stage of irAE development (62). Multiple studies demonstrated that the incidence of irAEs is significantly associated with the efficacy of ICIs (63), which can be partly explained by epitope spreading. Epitope spreading can also explain why different tumors treated with ICIs are associated with specific irAEs. For instance, in terms of antigenic epitopes, skin is the organ that shares $\mathrm{T}$ cell antigens with NSCLC only second to the lung and had the second highest incidence of irAEs (64). Berner et al. (64) identified nine shared T cell antigens in the lung tumor and skin in 25 patients with ICIs-induced skin toxicity. Besides, melanoma patients were more likely to develop mucosal and dermatological toxicities (65). Therefore, the use of ICIs should be prudent in melanoma or NSCLC patients with dermatological AIDs. Moreover, as mentioned above, some AIDs were inclined to worsen or result in devastating consequences during ICI therapy. Hence, in these specific situations, such as RA or GBS with active disease status, ICI applications should be avoided as much as possible. Furthermore, there have been many studies on biomarkers predicting irAEs (66), and increased autoantibody titers can also detect irAEs early $(30,67,68)$. Therefore, we do not think it is safe enough to use ICIs in patients with biomarkers that indicate a predisposition to specific irAEs in the same organs involved by their AID. For example, in a study of 26 melanoma patients treated with anti-CTLA-4, Firmicutes were associated with ICIs-induced colitis (69). Therefore, we should be more careful if we use ICIs in IBD patients with increased Firmicutes in the gut microbiome. However, those biomarkers predicting irAEs need to be validated prospectively.

To lower the risk of compromising ICIs efficacy, corticosteroids should be avoided as much as possible at the time of ICIs initiation. However, it is necessary to properly treat and control the preexisting AID before ICIs initiation. Therefore, Haanen et al. (70) proposed a two-step strategy for managing irAEs in AID patients. First, non-selective immunosuppressants (such as corticosteroids and mycophenolate mofetil) could be replaced by specific selective immunosuppressants (such as tocilizumab [anti-IL-6 receptor antibody], infliximab [antiTNF- $\alpha$ antibody], and vedolizumab [anti- $\alpha 4 \beta 7$ integrin antibody]) to control AIDs for a short period. Subsequently, combining ICIs with selective immunosuppressants could prevent the flare of preexisting AID. In a study of 87 patients who developed irAEs after treatment with nivolumab, clinical improvement was observed in $79 \%$ of 34 patients who received anti-IL-6 receptor antibody tocilizumab (71). On the aspect of efficacy, inhibition of IL- 6 has been reported to have synergistic anti-tumor activity when combined with ICIs in mouse models (65). Small series and several cases also have reported the safe and effective administration of ICIs in active AIDs under selective immunosuppressants (70). Theoretically, the two-step strategy recommended by Haanen et al. (70) seems to be valid when administrating ICIs in AID patients. Nevertheless, it is also important to note that the strategy is based on small-scale retrospective studies. Further studies are needed to confirm the effectiveness and safety of this strategy.

Intriguingly, irAEs can be prevented to some extent by modifying ICIs so that they are only active within the TME, or restricting their delivery to the TME. For example, ICIs can be loaded onto nanoparticles which possess intrinsic properties, like magnetism, and then be actively directed to accumulate in TME with the help of external devices (72). However, biomaterialbased cancer immunotherapies are mainly explored in animal studies, and deserve further validation in clinical trials.

\section{CONCLUSION}

There is no available prospective study on whether the efficacy and safety of ICIs in patients with cancer and preexisting AID differ from that in the general population. The results of the existing retrospective studies seemed to support that the efficacy of ICIs in patients with preexisting AID was comparable to that in the general population, and while the incidence of irAEs was higher, most irAEs were mild and manageable. However, there is a selection bias that most of the AID patients included in the 
existing retrospective studies were in stable disease status, patients' performance status was fair, and certain AIDs inclined to result in devastating consequences during ICI therapy may be under-represented. Given the existing data, preexisting AID may not be an absolute contraindication to ICI therapy. It is recommended to weigh the benefits and risks of immunotherapy in a multidisciplinary approach including rheumatologists based on a patient's AID category and severity, the patient's cancer type and prognosis, alternative treatment options, and the patient's intention. After that, ICIs could be administered with close monitoring of irAEs in patients with good performance status and mild AID status who could benefit from immunotherapy. Moreover, there are several strategies for preventing and meagering irAEs that can be taken when administrating ICIs in AID patients. Two phase I clinical trials of nivolumab in patients with AID and lung cancer (NCT03656627) or across tumor types (NCT03816345) are

\section{REFERENCES}

1. Fife BT, Bluestone JA. Control of peripheral T-cell tolerance and autoimmunity via the CTLA-4 and PD-1 pathways. Immunol Rev (2008) 224:166-82. doi: 10.1111/j.1600-065X.2008.00662.x

2. Krummel MF, Allison JP. CTLA-4 engagement inhibits IL-2 accumulation and cell cycle progression upon activation of resting T cells. J Exp Med (1996) 183:2533-40. doi: 10.1084/jem.183.6.2533

3. Boussiotis VA. Molecular and Biochemical Aspects of the PD-1 Checkpoint Pathway. N Engl J Med (2016) 375:1767-78. doi: 10.1056/NEJMra1514296

4. Martins F, Sofiya L, Sykiotis GP, Lamine F, Maillard M, Fraga M, et al. Adverse effects of immune-checkpoint inhibitors: epidemiology, management and surveillance. Nat Rev Clin Oncol (2019) 16:563-80. doi: 10.1038/s41571019-0218-0

5. Rose NR. Prediction and Prevention of Autoimmune Disease in the 21st Century: A Review and Preview. Am J Epidemiol (2016) 183:403-6. doi: 10.1093/aje/kwv292

6. Kennedy LC, Bhatia S, Thompson JA, Grivas P. Preexisting Autoimmune Disease: Implications for Immune Checkpoint Inhibitor Therapy in Solid Tumors. J Natl Compr Canc Netw (2019) 17:750-7. doi: 10.6004/ jnccn.2019.7310

7. Wieder T, Eigentler T, Brenner E, Röcken M. Immune checkpoint blockade therapy. J Allergy Clin Immunol (2018) 142:1403-14. doi: 10.1016/ j.jaci.2018.02.042

8. Khan SA, Pruitt SL, Xuan L, Gerber DE. Prevalence of Autoimmune Disease Among Patients With Lung Cancer: Implications for Immunotherapy Treatment Options. JAMA Oncol (2016) 2:1507-8. doi: 10.1001/ jamaoncol.2016.2238

9. Labrador-Horrillo M, Selva-O'Callaghan A. Cancer-associated Dermatomyositis: Does the PD-1 Checkpoint Pathway Play a Role? J Rheumatol (2018) 45:731-2. doi: 10.3899/jrheum.180007

10. Franks AL, Slansky JE. Multiple associations between a broad spectrum of autoimmune diseases, chronic inflammatory diseases and cancer. Anticancer Res (2012) 32:1119-36. doi: ar.iiarjournals.org/content/32/4/1119.long

11. Liang Y, Yang Z, Qin B, Zhong R. Primary Sjogren's syndrome and malignancy risk: a systematic review and meta-analysis. Ann Rheum Dis (2014) 73:1151-6. doi: 10.1136/annrheumdis-2013-203305

12. Mao S, Shen H, Zhang J. Systemic lupus erythematosus and malignancies risk. J Cancer Res Clin Oncol (2016) 142:253-62. doi: 10.1007/s00432-015-2032-0

13. Kazarian M, Laird-Offringa IA. Small-cell lung cancer-associated autoantibodies: potential applications to cancer diagnosis, early detection, and therapy. Mol Canc (2011) 10:33. doi: 10.1186/1476-4598-10-33

14. Dalmau J, Rosenfeld MR. Autoimmune encephalitis update. Neuro Oncol (2014) 16:771-8. doi: 10.1093/neuonc/nou030 ongoing. The results of the above two trials and more realworld retrospective studies would reach more instructive conclusions about the existing controversies.

\section{AUTHOR CONTRIBUTIONS}

Conception and design: HT and JZ. Literature review and analysis: HT and JZ. Drafting of the manuscript: HT and JZ. Supervision: JZ and CB. All authors contributed to the article and approved the submitted version.

\section{FUNDING}

This work is supported by a grant from CAMS Innovation Fund for Medical Sciences (no. 2016-I2M-1-001).

15. Menzies AM, Johnson DB, Ramanujam S, Atkinson VG, Wong ANM, Park JJ, et al. Anti-PD-1 therapy in patients with advanced melanoma and preexisting autoimmune disorders or major toxicity with ipilimumab. Ann Oncol (2017) 28:368-76. doi: 10.1093/annonc/mdw443

16. Zhang Q, Vignali DA. Co-stimulatory and Co-inhibitory Pathways in Autoimmunity. Immunity (2016) 44:1034-51. doi: 10.1016/j.immuni. 2016.04.017

17. Verma N, Burns SO, Walker LSK, Sansom DM. Immune deficiency and autoimmunity in patients with CTLA-4 (CD152) mutations. Clin Exp Immunol (2017) 190:1-7. doi: 10.1111/cei.12997

18. Tivol EA, Borriello F, Schweitzer AN, Lynch WP, Bluestone JA, Sharpe AH. Loss of CTLA-4 leads to massive lymphoproliferation and fatal multiorgan tissue destruction, revealing a critical negative regulatory role of CTLA-4. Immunity (1995) 3:541-7. doi: 10.1016/1074-7613(95)90125-6

19. Klocke K, Sakaguchi S, Holmdahl R, Wing K. Induction of autoimmune disease by deletion of CTLA-4 in mice in adulthood. Proc Natl Acad Sci USA (2016) 113:E2383-2392. doi: 10.1073/pnas.1603892113

20. Sharma R, Di Dalmazi G, Caturegli P. Exacerbation of Autoimmune Thyroiditis by CTLA-4 Blockade: A Role for IFN $\gamma$-Induced Indoleamine 2, 3-Dioxygenase. Thyroid (2016) 26:1117-24. doi: 10.1089/thy.2016.0092

21. Zamani MR, Aslani S, Salmaninejad A, Javan MR, Rezaei N. PD-1/PD-L and autoimmunity: A growing relationship. Cell Immunol (2016) 310:27-41. doi: 10.1016/j.cellimm.2016.09.009

22. Gotot J, Gottschalk C, Leopold S, Knolle PA, Yagita H, Kurts C, et al. Regulatory $\mathrm{T}$ cells use programmed death 1 ligands to directly suppress autoreactive B cells in vivo. Proc Natl Acad Sci USA (2012) 109:10468-73. doi: 10.1073/pnas.1201131109

23. Matsuda K, Miyoshi H, Hiraoka K, Hamada T, Yoshida S, Ishibashi Y, et al. Clinicopathological value of programmed cell death 1 (PD-1) and programmed cell death ligand 1 (PD-L1) expression in synovium of patients with rheumatoid arthritis. Clin Exp Med (2018) 18:487-94. doi: 10.1007/s10238-018-0515-4

24. Fife BT, Guleria I, Gubbels Bupp M, Eagar TN, Tang Q, Bour-Jordan H, et al. Insulin-induced remission in new-onset NOD mice is maintained by the PD1-PD-L1 pathway. J Exp Med (2006) 203:2737-47. doi: 10.1084/jem.20061577

25. Genovese MC, Becker JC, Schiff M, Luggen M, Sherrer Y, Kremer J, et al. Abatacept for rheumatoid arthritis refractory to tumor necrosis factor alpha inhibition. N Engl J Med (2005) 353:1114-23. doi: 10.1056/NEJMoa050524

26. Kähler KC, Eigentler TK, Gesierich A, Heinzerling L, Loquai C, Meier F, et al. Ipilimumab in metastatic melanoma patients with pre-existing autoimmune disorders. Cancer Immunol Immunother (2018) 67:825-34. doi: 10.1007/ s00262-018-2134-z

27. Johnson DB, Sullivan RJ, Ott PA, Carlino MS, Khushalani NI, Ye F, et al. Ipilimumab Therapy in Patients With Advanced Melanoma and Preexisting 
Autoimmune Disorders. JAMA Oncol (2016) 2:234-40. doi: 10.1001/ jamaoncol.2015.4368

28. Lee B, Wong A, Kee D, Neeson P, Shackleton M, McArthur G, et al. The use of ipilimumab in patients with rheumatoid arthritis and metastatic melanoma. Ann Oncol (2016) 27:1174-7. doi: 10.1093/annonc/mdw056

29. Gutzmer R, Koop A, Meier F, Hassel JC, Terheyden P, Zimmer L, et al. Programmed cell death protein-1 (PD-1) inhibitor therapy in patients with advanced melanoma and preexisting autoimmunity or ipilimumab-triggered autoimmunity. Eur J Cancer (2017) 75:24-32. doi: 10.1016/j.ejca.2016.12.038

30. Yoneshima Y, Tanaka K, Shiraishi Y, Hata K, Watanabe H, Harada T, et al. Safety and efficacy of PD-1 inhibitors in non-small cell lung cancer patients positive for antinuclear antibodies. Lung Cancer (2019) 130:5-9. doi: 10.1016/ j.lungcan.2019.01.014

31. Leonardi GC, Gainor JF, Altan M, Kravets S, Dahlberg SE, Gedmintas L, et al. Safety of Programmed Death-1 Pathway Inhibitors Among Patients With Non-Small-Cell Lung Cancer and Preexisting Autoimmune Disorders. J Clin Oncol (2018) 36:1905-12. doi: 10.1200/JCO.2017.77.0305

32. Loriot Y, Sternberg CN, Castellano D, Oosting SF, Dumez H, Huddart R, et al. Safety and efficacy of atezolizumab in patients with autoimmune disease: Subgroup analysis of the SAUL study in locally advanced/metastatic urinary tract carcinoma. Eur J Cancer (2020) 138:202-11. doi: 10.1016/ j.ejca.2020.07.023

33. Martinez Chanza N, Xie W, Issa M, Dzimitrowicz H, Tripathi A, Beuselinck B, et al. Safety and efficacy of immune checkpoint inhibitors in advanced urological cancers with pre-existing autoimmune disorders: a retrospective international multicenter study. J Immunother Cancer (2020) 8:e000538. doi: 10.1136/jitc-2020-000538

34. Danlos FX, Voisin AL, Dyevre V, Michot JM, Routier E, Taillade L, et al. Safety and efficacy of anti-programmed death 1 antibodies in patients with cancer and pre-existing autoimmune or inflammatory disease. Eur J Cancer (2018) 91:21-9. doi: 10.1016/j.ejca.2017.12.008

35. Cortellini A, Buti S, Santini D, Perrone F, Giusti R, Tiseo M, et al. Clinical Outcomes of Patients with Advanced Cancer and Pre-Existing Autoimmune Diseases Treated with Anti-Programmed Death-1 Immunotherapy: A RealWorld Transverse Study. Oncologist (2019) 24:e327-37. doi: 10.1634/ theoncologist.2018-0618

36. Tison A, Quéré G, Misery L, Funck-Brentano E, Danlos FX, Routier E, et al. Safety and Efficacy of Immune Checkpoint Inhibitors in Patients With Cancer and Preexisting Autoimmune Disease: A Nationwide, Multicenter Cohort Study. Arthritis Rheumatol (2019) 71:2100-11. doi: 10.1002/art.41068

37. Richter MD, Pinkston O, Kottschade LA, Finnes HD, Markovic SN, Thanarajasingam U. Brief Report: Cancer Immunotherapy in Patients With Preexisting Rheumatic Disease: The Mayo Clinic Experience. Arthritis Rheumatol (2018) 70:356-60. doi: 10.1002/art.40397

38. Kaur A, Doberstein T, Amberker RR, Garje R, Field EH, Singh N. Immunerelated adverse events in cancer patients treated with immune checkpoint inhibitors: A single-center experience. Med (Baltimore) (2019) 98:e17348. doi: 10.1097/MD.0000000000017348

39. Abu-Sbeih H, Faleck DM, Ricciuti B, Mendelsohn RB, Naqash AR, Cohen JV, et al. Immune Checkpoint Inhibitor Therapy in Patients With Preexisting Inflammatory Bowel Disease. J Clin Oncol (2020) 38:576-83. doi: 10.1200/ JCO.19.01674

40. Braga Neto MB, Ramos GP, Loftus EVJr., Faubion WA, Raffals LE. Use of Immune Checkpoint Inhibitors in Patients With Pre-established Inflammatory Bowel Diseases: Retrospective Case Series. Clin Gastroenterol Hepatol Off Clin Pract J Am Gastroenterol Assoc (2020). doi: 10.1016/ j.cgh.2020.06.031

41. Efuni E, Cytryn S, Boland P, Niewold TB, Pavlick A, Weber J, et al. Risk of Toxicity After Initiating Immune Checkpoint Inhibitor Treatment in Patients With Rheumatoid Arthritis. J Clin Rheumatol Pract Rep Rheumatic Musculoskeletal Dis (2020). doi: 10.1097/RHU.0000000000001314

42. Hodi FS, Chiarion-Sileni V, Gonzalez R, Grob JJ, Rutkowski P, Cowey CL, et al. Nivolumab plus ipilimumab or nivolumab alone versus ipilimumab alone in advanced melanoma (CheckMate 067): 4-year outcomes of a multicentre, randomised, phase 3 trial. Lancet Oncol (2018) 19:1480-92. doi: 10.1016/S1470-2045(18)30700-9

43. Schachter J, Ribas A, Long GV, Arance A, Grob JJ, Mortier L, et al. Pembrolizumab versus ipilimumab for advanced melanoma: final overall survival results of a multicentre, randomised, open-label phase 3 study (KEYNOTE-006). Lancet (2017) 390:1853-62. doi: 10.1016/S0140-6736(17) 31601-X

44. Haanen J, Carbonnel F, Robert C, Kerr KM, Peters S, Larkin J, et al. Management of toxicities from immunotherapy: ESMO Clinical Practice Guidelines for diagnosis, treatment and follow-up. Ann Oncol (2018) 29: iv264-6. doi: 10.1093/annonc/mdy162

45. Puzanov I, Diab A, Abdallah K, Bingham CO,3, Brogdon C, Dadu R, et al. Managing toxicities associated with immune checkpoint inhibitors: consensus recommendations from the Society for Immunotherapy of Cancer (SITC) Toxicity Management Working Group. J Immunother Cancer (2017) 5:95. doi: 10.1186/s40425-017-0300-z

46. Thompson JA, Schneider BJ, Brahmer J, Andrews S, Armand P, Bhatia S, et al. NCCN Guidelines Insights: Management of Immunotherapy-Related Toxicities, Version 1.2020. J Natl Compr Canc Netw (2020) 18:230-41. doi: 10.6004/jnccn.2020.0012

47. Robert C, Long GV, Brady B, Dutriaux C, Maio M, Mortier L, et al. Nivolumab in previously untreated melanoma without BRAF mutation. $N$ Engl J Med (2015) 372:320-30. doi: 10.1056/NEJMoa1412082

48. Weber JS, D'Angelo SP, Minor D, Hodi FS, Gutzmer R, Neyns B, et al. Nivolumab versus chemotherapy in patients with advanced melanoma who progressed after anti-CTLA-4 treatment (CheckMate 037): a randomised, controlled, open-label, phase 3 trial. Lancet Oncol (2015) 16:375-84. doi: 10.1016/S1470-2045(15)70076-8

49. Ribas A, Puzanov I, Dummer R, Schadendorf D, Hamid O, Robert C, et al. Pembrolizumab versus investigator-choice chemotherapy for ipilimumabrefractory melanoma (KEYNOTE-002): a randomised, controlled, phase 2 trial. Lancet Oncol (2015) 16:908-18. doi: 10.1016/S1470-2045(15)00083-2

50. Ricciuti B, Dahlberg SE, Adeni A, Sholl LM, Nishino M, Awad MM. Immune Checkpoint Inhibitor Outcomes for Patients With Non-Small-Cell Lung Cancer Receiving Baseline Corticosteroids for Palliative Versus Nonpalliative Indications. J Clin Oncol (2019) 37:1927-34. doi: 10.1200/ JCO.19.00189

51. Arbour KC, Mezquita L, Long N, Rizvi H, Auclin E, Ni A, et al. Impact of Baseline Steroids on Efficacy of Programmed Cell Death-1 and Programmed Death-Ligand 1 Blockade in Patients With Non-Small-Cell Lung Cancer. J Clin Oncol (2018) 36:2872-8. doi: 10.1200/JCO.2018.79.0006

52. Xie W, Huang H, Xiao S, Fan Y, Deng X, Zhang Z. Immune checkpoint inhibitors therapies in patients with cancer and preexisting autoimmune diseases: A meta-analysis of observational studies. Autoimmun Rev (2020) 19:102687. doi: 10.1016/j.autrev.2020.102687

53. Ramos-Casals M, Brahmer JR, Callahan MK, Flores-Chávez A, Keegan N, Khamashta MA, et al. Immune-related adverse events of checkpoint inhibitors. Nat Rev Dis Primers (2020) 6:38. doi: 10.1038/s41572-020-0160-6

54. van den Berg B, Walgaard C, Drenthen J, Fokke C, Jacobs BC, van Doorn PA. Guillain-Barré syndrome: pathogenesis, diagnosis, treatment and prognosis. Nat Rev Neurol (2014) 10:469-82. doi: 10.1038/nrneurol.2014.121

55. Gu-Trantien C, Willard-Gallo K. PD-1(hi)CXCR5(-)CD4(+) T(FH) Cells Play Defense in Cancer and Offense in Arthritis. Trends Immunol (2017) 38:875-8. doi: 10.1016/j.it.2017.10.003

56. El Osta B, Hu F, Sadek R, Chintalapally R, Tang SC. Not all immunecheckpoint inhibitors are created equal: Meta-analysis and systematic review of immune-related adverse events in cancer trials. Crit Rev Oncol Hematol (2017) 119:1-12. doi: 10.1016/j.critrevonc.2017.09.002

57. Nishimura H, Nose M, Hiai H, Minato N, Honjo T. Development of lupuslike autoimmune diseases by disruption of the PD-1 gene encoding an ITIM motif-carrying immunoreceptor. Immunity (1999) 11:141-51. doi: 10.1016/ S1074-7613(00)80089-8

58. Hollinger MK, Giudice V, Cummings NA, Rivell G, Zhang H, Kajigaya S, et al. $\mathrm{PD}-1$ deficiency augments bone marrow failure in a minor-histocompatibility antigen mismatch lymphocyte infusion model. Exp Hematol (2018) 62:17-23. doi: 10.1016/j.exphem.2018.03.001

59. Caturegli P, Di Dalmazi G, Lombardi M, Grosso F, Larman HB, Larman T, et al. Hypophysitis Secondary to Cytotoxic T-Lymphocyte-Associated Protein 4 Blockade: Insights into Pathogenesis from an Autopsy Series. Am J Pathol (2016) 186:3225-35. doi: 10.1016/j.ajpath.2016.08.020

60. Iwama S, De Remigis A, Callahan MK, Slovin SF, Wolchok JD, Caturegli P. Pituitary expression of CTLA-4 mediates hypophysitis secondary to 
administration of CTLA-4 blocking antibody. Sci Transl Med (2014) 6:230ra245. doi: 10.1126/scitranslmed.3008002

61. Abdel-Wahab N, Shah M, Lopez-Olivo MA, Suarez-Almazor ME. Use of Immune Checkpoint Inhibitors in the Treatment of Patients With Cancer and Preexisting Autoimmune Disease: A Systematic Review. Ann Intern Med (2018) 168:121-30. doi: 10.7326/M17-2073

62. Yang H, Yao Z, Zhou X, Zhang W, Zhang X, Zhang F. Immune-related adverse events of checkpoint inhibitors: Insights into immunological dysregulation. Clin Immunol (2020) 213:108377. doi: 10.1016/ j.clim.2020.108377

63. Petrelli F, Grizzi G, Ghidini M, Ghidini A, Ratti M, Panni S, et al. Immunerelated Adverse Events and Survival in Solid Tumors Treated With Immune Checkpoint Inhibitors: A Systematic Review and Meta-Analysis. J Immunother (Hagerstown Md 1997) (2020) 43:1-7. doi: 10.1097/CJI.0000 000000000300

64. Berner F, Bomze D, Diem S, Ali OH, Fässler M, Ring S, et al. Association of Checkpoint Inhibitor-Induced Toxic Effects With Shared Cancer and Tissue Antigens in Non-Small Cell Lung Cancer. JAMA Oncol (2019) 5:1043-7. doi: 10.1001/jamaoncol.2019.0402

65. Esfahani K, Elkrief A, Calabrese C, Lapointe R, Hudson M, Routy B, et al. Moving towards personalized treatments of immune-related adverse events. Nat Rev Clin Oncol (2020) 17:504-15. doi: 10.1038/s41571020-0352-8

66. von Itzstein MS, Khan S, Gerber DE. Investigational Biomarkers for Checkpoint Inhibitor Immune-Related Adverse Event Prediction and Diagnosis. Clin Chem (2020) 66:779-93. doi: 10.1093/clinchem/hvaa081

67. Tahir SA, Gao J, Miura Y, Blando J, Tidwell RSS, Zhao H, et al. Autoimmune antibodies correlate with immune checkpoint therapyinduced toxicities. Proc Natl Acad Sci U States A (2019) 116:22246-51. doi: 10.1073/pnas.1908079116
68. Gowen MF, Giles KM, Simpson D, Tchack J, Zhou H, Moran U, et al. Baseline antibody profiles predict toxicity in melanoma patients treated with immune checkpoint inhibitors. J Trans Med (2018) 16:82. doi: 10.1186/s12967-018$1452-4$

69. Chaput N, Lepage P, Coutzac C, Soularue E, Le Roux K, Monot C, et al. Baseline gut microbiota predicts clinical response and colitis in metastatic melanoma patients treated with ipilimumab. Ann Oncol (2017) 28:1368-79. doi: $10.1093 / \mathrm{annonc} / \mathrm{mdx} 108$

70. Haanen J, Ernstoff MS, Wang Y, Menzies AM, Puzanov I, Grivas P, et al. Autoimmune diseases and immune-checkpoint inhibitors for cancer therapy: review of the literature and personalized risk-based prevention strategy. Ann Oncol (2020) 31:724-44. doi: 10.1016/j.annonc.2020.03.285

71. Stroud CR, Hegde A, Cherry C, Naqash AR, Sharma N, Addepalli S, et al. Tocilizumab for the management of immune mediated adverse events secondary to PD-1 blockade. J Oncol Pharm Pract Off Publ Int Soc Oncol Pharm Practitioners (2019) 25:551-7. doi: 10.1177/1078155217745144

72. Hu D, Zhang W, Tang J, Zhou Z, Liu X, Shen Y. Improving safety of cancer immunotherapy via delivery technology. Biomaterials (2020) 265:120407. doi: 10.1016/j.biomaterials.2020.120407

Conflict of Interest: The authors declare that the review was conducted in the absence of any commercial or financial relationships that could be construed as a potential conflict of interest.

Copyright $\odot 2021$ Tang, Zhou and Bai. This is an open-access article distributed under the terms of the Creative Commons Attribution License (CC BY). The use, distribution or reproduction in other forums is permitted, provided the original author(s) and the copyright owner(s) are credited and that the original publication in this journal is cited, in accordance with accepted academic practice. No use, distribution or reproduction is permitted which does not comply with these terms. 